\title{
Projeto do Dicionário Winnicott \\ Winnicott Dictionary Project
}

\section{Zeljko Loparic \\ SBPW/IWA \\ E-mail: loparicz@uol.com.br}

Resumo: Não raramente as contribuições de Winnicott à psicanálise são consideradas uma simples extensão da psicanálise freudiana, ampliada por M. Klein. Essa visão sobre Winnicott foi contestada, há bastante tempo, por vários pesquisadores. Já nos anos 1950, Masud Khan sustentou a tese de que Winnicott introduziu mudanças revolucionárias no pensamento analítico e na prática contemporâneos. O fato de Winnicott ter operado mudanças revolucionárias, criando um novo "paradigma" ou uma nova "matriz" na psicanálise, foi reconhecido, a partir dos anos 1980, com mais força ainda, embora nem sempre com a referência explícita a Kuhn. No Brasil, pesquisadores vinculados ao Grupo de Pesquisa em Filosofia e Práticas Psicoterápicas (Unicamp) levaram em conta, de maneira sistemática e articulada, todos os elementos da teoria kuhniana dos paradigmas e das revoluções científicas, devidamente adaptadas ao caso da psicanálise. O presente Projeto parte da constatação de que não é mais necessário debater se uma revolução na psicanálise estava em pauta para Winnicott, nem mesmo se ele deu passos nessa direção, visto que isso já está suficientemente provado. É preciso estudar melhor os motivos da sua pesquisa revolucionária e os resultados alcançados. Para esse propósito, o presente Projeto do Dicionário Winnicott - que se propõe a reconstruir, de forma exaustiva e conceitualmente articulada, o léxico do paradigma winnicottiano - espera trazer uma contribuição decisiva.

Palavras-chave: Dicionário Winnicott, revolução winnicottiana, paradigma winnicottiano, Sociedade Brasileira de Psicanálise Winnicottiana, Escola Winnicottiana de São Paulo.

\begin{abstract}
There are those who maintain that Winnicott has not brought any new and conclusive contribution to the psychoanalytic theory. Some readers still consider his contributions as a simple extension of Freudian psycho-analytical theory, which was expanded by M. Klein. This point of view regarding Winnicott has been contested, however, by many of his researchers. During the fifties Masud Khan sustained the thesis that Winnicott has made revolutionary changes in contemporary psychoanalytic thought and practice. The fact that Winnicott has produced revolutionary changes, creating a "new paradigm" or a "new matrix" in psycho-analysis, was acknowledged since the eighties, with even more strength, although not always with the explicit reference to Kuhn. In Brazil, researchers linked to the Research Group in Philosophy and
\end{abstract}


Psychotherapeutic Practices (Unicamp) took into account, in a systematic and articulated way, all the elements of Kuhn's theory concerning paradigms and scientific revolutions, duly adapted to the psycho-analysis. This Project starts from the assumption that it is no longer needed to debate if Winnicott promoted a revolution in the Psycho-analytic theory, nor if he followed in that direction, since this has already been proved. What is necessary is to improve studies concerning the motives of his revolutionary research and the obtained results. For this purpose, the present Winnicott Dictionary Project intends to reconstruct, in an exhaustive way and with an articulated conceptualization, the lexicon of the winnicottian paradigm.

Key-words: Winnicott Dictionary, Winnicottian revolution, Winnicottian paradigm. Brazilian Society for Winnicottian Psychoanalysis, São Paulo Winnicottian School.

Adultos maduros levam vitalidade ao que é antigo, velho e ortodoxo, recriando essas coisas depois de tê-las destruído.

Winnicott

\section{As mudanças revolucionárias de Winnicott}

Winnicott foi e continua sendo reconhecido por muitos como um clínico genial, que, contudo, não trouxe uma contribuição nova e decisiva à teoria psicanalítica. Não raramente as suas contribuições à psicanálise são consideradas uma simples extensão da psicanálise freudiana, ampliada por M. Klein. Essa visão sobre Winnicott foi contestada, há bastante tempo, por vários de seus interlocutores. Já nos anos 1950, Masud Khan sustentou a teses de que a distinção conceitual e terminológica winnicottiana entre "necessidades do ego" e "necessidades do id" constitui "uma mudança revolucionária de ênfase no pensamento analítico e na prática contemporâneos" (Winnicott, 1958, p. XV). Em 1968, M. Balint enalteceu Winnicott como “o mais versátil criador" de novos termos teóricos da psicanálise (Balint, 1968/1992, p. 168) e sugeriu que uma "nova linguagem" própria da "Escola de manejo", diferente das escolas freudiana e kleiniana, poderia ser desenvolvida "sob a influência das ideias de Winnicott" (p. 116). Em 1975, A. Green chegou a afirmar que as contribuições de Winnicott colocam em pauta a pergunta pelo futuro da psicanálise, a qual, não raramente, permanece apegada a posições teóricas e clínicas ultrapassadas e se furta, dessa maneira, à necessidade de, diante de impasses teóricos e clínicos, buscar alternativas, renovar-se periodicamente, estender o seu alcance, submeter seus conceitos a modificações radicais, compromissada, como era em Freud, com a autocrítica (Green, 1975/2013, p. 193). No seu livro Boundary and Space (1981), 
Davis e Wallbridge sublinham que a novidade da contribuição de Winnicott consistia na introdução de uma "outra linguagem" para fenômenos da vida humana e da clínica até então não nomeados pela psicanálise (p. 116).

Pouco mais tarde, Greenberg e Mitchell (1983), continuando as abordagens anteriores de Modell (1968) e Lifton (1976), entre outros, fizeram um avanço decisivo ao proporem explicitamente uma leitura de Winnicott segundo a qual a sua contribuição à psicanálise consistia em uma revolução do tipo kuhniano e não em uma simples ampliação ou atualização do paradigma freudiano. O uso de Kuhn é justificado da seguinte maneira:

Estamos sugerindo que a abordagem de Kuhn do desenvolvimento das ideias científicas e a sua definição dos modelos como compromissos metafísicos são altamente aplicáveis à história do pensamento psicanalítico e constituem um modo útil de abordagem dos diferentes estágios da construção de teorias. (Greenberg \& Mitchell, 1983, p. 19)

Num texto escrito em 1984, Rycroft recorda a sua formação na BPS dividida em annafreudianos, kleinianos e "independentes", e relata:

Foi só então [depois de ter-se formado como analista e começado a clinicar] que pude chegar a perceber que as divergências de opinião sobre a teoria entre os três grupos eram realmente diferenças de substância; que Anna Freud, Melanie Klein e D. W. Winnicott tinham realmente concepções diferentes da natureza humana, da saúde, de como uma análise deve ser conduzida e o que constitui uma análise bem-sucedida. (Rycroft, 1985, p. 120)

Mais recentemente, a leitura da contribuição winnicottiana à história da psicanálise a partir da perspectiva kuhniana foi adotada por Joyce McDougall (1997, p. 226) e, em 2000, Mitchell reafirmou as teses que defendeu em 1983 com Greenberg.

O fato de Winnicott ter operado "mudanças revolucionárias", criando um novo "paradigma" ou uma nova "matriz" na psicanálise, foi reconhecido, a partir dos anos 1980, com mais força ainda, embora nem sempre com a referência explícita a Kuhn, por A. Phillips (1988), P. C. Horton, H. Gerwith e K. J. Kreutter (1988), J. Hodges (1989), T. H. Ogden (2002), D. Widlöcher (2006/2013), K. Wright (2009/2013), R. Roussillon (2010), Caldwell e Joyce (2011) e N. Thompson (2013). Ogden, por exemplo, sustenta 
que Winnicott introduziu um grande número de "revoluções silenciosas" na psicanálise, tendo "revolucionado", em particular, a concepção de "quadro analítico" (2002, p. 213). Por sua parte, Caldwell e Joyce observam que Winnicott foi "figura central" na elaboração “da teoria britânica de relações objetais, a qual, mesmo tendo raízes freudianas, revolucionou a psicanálise moderna" (2011, p. 1).

O conceito kuhniano de mudança paradigmática foi usado também para caracterizar as contribuições de outros autores. Os primeiros a assim proceder de maneira sistemática foram Greenberg e Mitchell, na obra citada anteriormente. Antes deles, Heinz Kohut se inspirou em Kuhn para dar à sua própria concepção de distúrbios narcísicos da personalidade o sentido de uma modificação da psicanálise semelhante a uma "mutação" biológica, dizendo tratar-se de uma contribuição "que fornece acesso a um inteiramente novo aspecto da realidade" (1975, p. 299) e que conjuga uma nova técnica revolucionária com uma nova teoria de explicação, ambas tendo um valor paradigmático.

Em 1992, Paul H. Ornstein - na sua introdução às ideias seminais de Michael Balint sobre o processo de tratamento psicanalítico - observa que essas ideias foram recebidas no campo da psicanálise de duas maneiras opostas. Um primeiro grupo de leitores considerou que as modificações técnicas de Balint eram aceitáveis apenas a título de "parâmetros" clínicos a serem interpretados e, por último, descartados, mas "não como "mudança do paradigma básico" freudiano, o qual já teria sido devidamente ampliado pela psicologia do ego (Ornstein, 1992, p. XVIII). Outro grupo recusou essa redução e buscou na obra de Balint uma "melhor solução para seus problemas clínicos", superando dessa forma "a pressão grupal e o estrangulamento [stranglehold] pelo paradigma dominante" (p. XVIII). Na opinião desse grupo, o "conserto" e a expansão da psicanálise freudiana pela psicologia do ego não era suficiente, precisando a psicanálise passar por “uma revisão mais importante” (p. XIX).

\section{O Projeto de criação do Dicionário Winnicott: abrangência internacional}

Nos anos 1980, iniciei, de forma independente e sistemática - seguindo uma linha de pesquisa elaborada para essa finalidade e aceita por um grupo de pesquisadores da 
Unicamp e da PUC-SP que se tornou o Grupo de Pesquisa em Filosofia e Práticas Psicoterápicas (GrupoFPP) do CNPq -, uma leitura kuhniana da história da psicanálise e, em particular, da contribuição de Winnicott. Em que pesem as tentativas anteriores desse tipo de reconstrução da história e da estrutura da psicanálise winnicottiana, esse grupo de pesquisa, atualmente ativo também na Sociedade Brasileira de Psicanálise Winnicottiana (SBPW), foi o primeiro a levar em conta e articular todos os elementos da teoria kuhniana dos paradigmas e das revoluções científicas, devidamente adaptada ao caso da psicanálise, razão pela qual passou a ser chamada de "Escola Winnicottiana de São Paulo". Em particular, o conceito kuhniano de paradigma não é reduzido, como em Greenberg e Mitchell, aos compromissos metafísicos, sendo usado em toda sua complexidade.

A linha de pesquisa mencionada - articulada adicionalmente em colaboração com Elsa Oliveira Dias - é fundamentada nas seguintes teses principais, inspiradas em Kuhn e em resultados de pesquisas que vêm sendo realizadas ao longo de anos:

1) A história da psicanálise pode ser analisada e articulada em termos da teoria kuhniana das crises científicas, revoluções científicas e de novos paradigmas.

2) A contribuição de Winnicott consiste na elaboração de um novo paradigma da psicanálise, que representa uma modificação revolucionária do paradigma freudiano e permanece sem paralelo na história dessa disciplina.

3) A pesquisa revolucionária de Winnicott foi motivada pela crise da psicanálise freudiana, devido ao acúmulo de problemas não solúveis (anomalias), em especial as dificuldades relativas aos bebês na fase de lactância, emparelhadas, por Winnicott, às dos psicóticos.

4) A parte central do corpo teórico da psicanálise winnicottiana é a teoria do amadurecimento, que contém, como um de seus componentes, a teoria da sexualidade, e que serve de quadro geral para o estudo das necessidades fundamentais de um recém-nascido, as quais se originam principalmente da tendência inata para a integração, constitutiva da natureza humana, e das condições ambientais que favorecem, ou falham em favorecer, a realização dessa tendência.

5) A teoria do amadurecimento é o horizonte para o estudo da natureza e para a etiologia dos distúrbios a cargo da psicanálise, concebidos como distúrbios do processo de amadurecimento. 
6) A teoria do amadurecimento é, igualmente, a base e o guia da clínica winnicottiana.

7) O componente ontológico do paradigma winnicottiano não é a metapsicologia freudiana; esta é substituída por um horizonte filosófico que pode ser articulado, ainda que apenas parcialmente, com base nos elementos do existencialismo e da analítica existencial de Heidegger.

8) A situação exemplar da psicanálise winnicottiana não é mais a situação edípica - a "criança na cama da mãe" -, mas a relação mãe-bebê - o "bebê no colo da mãe".

9) A teoria de amadurecimento contém uma teoria da socialização que permite aplicar a psicanálise winnicottiana:

a) ao estudo do processo de socialização e da estrutura da sociedade, da ordem democrática, da história da humanidade a da vida social em geral;

b) à clarificação da natureza e à etiologia dos distúrbios da socialização e da ordem social;

c) à prevenção e ao tratamento desses problemas, possibilitando a colaboração direta com as atividades realizadas pelos serviços sociais públicos e privados em diferentes áreas.

Com bases nesses resultados, surgiu a proposta de construir o Dicionário Winnicott, trabalho essencial para a caracterização do paradigma winnicottiano. O projeto do Dicionário Winnicott inscreve-se numa das principais tradições de historiografia e filosofia da ciência, posta em evidência por Thomas S. Kuhn no seu livro A estrutura das revoluções científicas (1962). Ao sustentar a concepção de que a ciência não busca a verdade última sobre o mundo em si, mas desenvolve a atividade de resolução de problemas de certos tipos relativos ao mundo tal como ele nos é dado, Kuhn retoma uma posição já presente na Antiguidade ("salvar os fenômenos"), e rearticulada posteriormente por filósofos e cientistas de primeira linha, entre eles Descartes, Kant e Carnap. A atualidade de Kuhn é atestada pela pesquisa contínua e consistente sobre a sua obra (veja, por exemplo, Bird, 2000, e Nickles, 2003). Ao escrever a introdução à edição dos 50 anos de $A$ estrutura, Ian Hacking afirmou: "esse livro realmente mudou 'a imagem da ciência que hoje nos domina'. Para sempre” (Hacking, 2012, p. XXXVII).

Além disso, o Dicionário Winnicott nasce em um contexto de reconhecimento internacional crescente da interpretação kuhniana da psicanálise winnicottiana proposta 
pela Escola Winnicottiana de São Paulo e de desenvolvimentos institucionais recentes favoráveis a essa iniciativa.

Em 2007, na introdução à segunda edição do seu aclamado dicionário The Language of Winnicott, Jan Abram usa a linguagem de Kuhn para dizer que Winnicott, ao mesmo tempo em que continua Freud e Klein, introduziu "uma genuína mudança paradigmática [paradigm shift] no pensamento psicanalítico" (p. 5). Um ano depois, num artigo de 2008, Abram me cita (Loparic, 2002 e 2006) para assinalar que, segundo alguns autores, "a psicanálise winnicottiana constitui uma mudança paradigmática [paradigm shift]" (Abram, 2013, p. 94). Mais recentemente ainda, na primeira frase da introdução à sua coletânea Donald Winnicott Today, publicada em 2013, Abram escreveu: “O principal objetivo deste volume é demonstrar que a contribuição de Winnicott constitui uma revolução de maior importância na psicanálise”. Em apoio a essa afirmação ousada, Abram cita Kuhn e o meu artigo sobre o esboço do paradigma winnicottiano, reeditado na mesma coletânea. Ela escreve:

A palavra "revolução" refere-se à obra de Thomas Kuhn no seu livro Estrutura das revoluções científicas (1970, 2. Edição). No capítulo 4 [da coletânea editada por Jan Abram em 2013] Loparic se propõe a examinar a evolução das descobertas de Winnicott e a aplicá-las à teoria kuhniana das revoluções científicas. Loparic faz ver a extensão da mudança realizada por Winnicott na matriz psicanalítica simbólica de Freud. (Abram, 2013, p. 22)

No seu resumo do meu texto, Abram afirma:

A tese de que a obra de Winnicott constitui uma revolução científica foi comprovada por Zeljko Loparic no capítulo 4. Apoiada na teoria de Thomas Kuhn da "estrutura das revoluções científicas", essa perspectiva oferece um argumento claro e convincente que mostra como Winnicott adiciona ao paradigma edípico de Freud o "paradigma do bebê-no-colo-da-mãe". Loparic sugere que isso foi a solução para a "crise" científica de Winnicott que surgiu quando ele deu-se conta de que os bebês podiam realmente ficar doentes. [...] Loparic demonstra que a posição teórica final de Winnicott, diferente da de Freud, está relacionada aos seus pontos de partida diferentes. Enquanto o paradigma edípico de Freud emergiu do trabalho deste com os casos de histeria, Winnicott, pediatra e analista de crianças, foi confrontado com o problema do bebê doente no colo da mãe. Essa 
é, no essencial, a mudança paradigmática que explica a diferença entre a psicanálise freudiana edípica, triangular, de "três corpos", e a winnicottiana da relação mãe-bebê, dual ou de "dois corpos".

Loparic aborda de modo abrangente o caráter específico da pesquisa revolucionária de Winnicott e completa sua análise crítica com uma discussão com os críticos de Winnicott (bem como com aqueles que fazem uso fantasioso da sua obra). Loparic enfatiza que, seguindo tanto Darwin como Freud, Winnicott era em primeiro lugar um cientista leal ao método científico. A fim de ilustrar esse ponto, Loparic cita a metodologia imensamente valiosa das consultas terapêuticas de Winnicott, que exemplificam sua dedicação à pesquisa científica. (Abram, 2013, pp. 5-6)

Num artigo publicado também em 2013, no número especial da Psychoanalytic Inquiry dedicado a Winnicott, Ofra Eshel defendeu de modo explícito, citando em apoio trabalhos de membros da Escola Winnicottiana de São Paulo, uma abordagem kuhniana das mudanças que Winnicott introduziu na psicanálise. A autora trouxe novos argumentos a favor dessa posição no seu trabalho "On Winnicott's paradigm shift - Clinical psychoanalysis at its most formative edge" (Sobre a mudança paradigmática de Winnicott - A psicanálise clínica na sua ponta mais formativa), apresentado em 2015 no I Congresso Winnicott Internacional da International Winnicott Association (IWA), a ser publicado em Loparic, 2016. Essa coletânea conterá ainda outros trabalhos que aprofundam o estudo da revolução winnicottiana.

Contudo, mais significativo ainda que esse reconhecimento internacional das teses que, desde 1995, tornaram-se referência central para os trabalhos do Grupo de Pesquisa em Filosofia e Práticas Psicoterápicas (GrupoFPP) e, posteriormente, da Sociedade Brasileira de Psicanálise Winnicottiana (SBPW), é o fato de o próprio Winnicott, num texto escrito em 1970-71, portanto, já no final da sua vida, ter defendido explicitamente a necessidade de uma revolução na psicanálise. A frase que resume a sua proposta merece ser citada: "Estou pleiteando uma espécie de revolução no nosso trabalho. Vamos reexaminar o que estamos fazendo" (apud Abram, 2013, pp. 312-313).

Sendo assim, o presente Projeto parte da constatação de que não é mais necessário debater se uma revolução na psicanálise estava em pauta para Winnicott, nem mesmo se ele deu passos nessa direção. O que é preciso, isso sim, é estudar melhor os motivos da sua pesquisa revolucionária e os resultados alcançados. Para esse propósito, o presente Projeto do Dicionário Winnicott - que se propõe a reconstruir, de forma exaustiva e 
conceitualmente articulada, o léxico do paradigma winnicottiano - espera trazer uma contribuição decisiva.

\section{A linguagem da psicanálise winnicottiana}

Baseado na linha de pesquisa acima resumida, explicitei, em vários textos, os principais elementos da estrutura do paradigma winnicottiano, sempre em comparação com os elementos do paradigma freudiano.

Elsa Oliveira Dias, por sua vez, após pesquisar toda a obra de Winnicott (além da de seus comentadores), reunindo e articulando os elementos que permitissem uma apresentação unitária do seu pensamento, dedicou-se ao estudo detalhado do eixo central da psicanálise winnicottiana: a teoria do amadurecimento. A articulação final dessa teoria (que continua em boa parte desconhecida em vários grupos psicanalíticos, nacionais e internacionais, inclusive entre os que se interessam por Winnicott) revelou-se, verdadeiramente, tal como o próprio autor afirmou, o backbone, (a espinha dorsal) do seu pensamento e, em particular, de sua teoria dos distúrbios psíquicos.

Sobre esse horizonte, vários trabalhos desdobraram seus temas específicos, seja a respeito de elementos estruturais ou fases da temporalização da natureza humana - a lactância, a infância, a adolescência, o conceito de ambiente, a criatividade, a sexualidade, os aspectos dissociados na formação da identidade de gênero, a constituição psicossomática do eu -, seja relacionados à nova formulação winnicottiana, também baseada na teoria do amadurecimento, sobre a natureza e a etiologia dos distúrbios psíquicos - entre eles, as psicoses, os transtornos psicossomáticos, o autismo, a depressão, as paranoias, a tendência antissocial e a neurose. Outros ainda foram dedicados aos novos procedimentos clínicos que decorrem do paradigma winnicottiano, relevantes para o tratamento de vários desses distúrbios: o novo sentido da transferência e da interpretação, o manejo, o uso terapêutico da falha do analista e as consultas terapêuticas. Não faltaram trabalhos dedicados à aplicação da psicanálise winnicottiana a problemas não estritamente clínicos, tais como rotinas neonatais ou adoção.

Mais recentemente, aspectos específicos da teoria do amadurecimento foram tomados para estudos aprofundados e significativamente ampliados, em particular pela introdução dos temas do pai - que permitiu uma grande expansão sobre a problemática do concernimento e, portanto, sobre as questões relativas à aquisição da capacidade para 
a depressão simples - e da família. Essa extensão da temática do amadurecimento permitiu reconstituir a redescrição winnicottiana das relações triangulares com base genital (o Édipo) e avançar pelo estudo da adolescência, do processo de socialização, além de problemas relativos ao envelhecimento. Outros aportes foram realizados na direção de aplicar a teoria winnicottiana do amadurecimento na prevenção dos distúrbios psíquicos e no tratamento não estritamente psicanalítico-clínico de problemas sociais e de saúde.

A linguagem do paradigma freudiano foi estudada de forma exemplar no Vocabulário de Psicanálise por J. Laplanche e J.-B. Pontalis (1967). Contudo, uma das limitações dessa obra é a restrição à linguagem técnico-clínica de Freud. Essa limitação é em parte superada pelo Dictionaire de la psychanalyse de E. Roudinesco e M. Plon (nova edição, 1997), que é uma contribuição adicional apreciável na mesma direção, mas que também não cobre por completo o léxico freudiano.

Até o presente momento, a linguagem da psicanálise winnicottiana foi dicionarizada apenas por Jan Abram em The Language of Winnicott, publicado em 1996. Baseado em um dos mais detalhados estudos da totalidade da obra de Winnicott realizado até aquela data, acompanhando o desenvolvimento das ideias de Winnicott e as diferenças entre suas concepções e as dos autores centrais da psicanálise tradicional (Freud, Klein), esse dicionário, universalmente aplaudido, foi um primeiro passo importante num território até então inexplorado. Contudo, considerado à luz de resultados mais recentes no campo de estudos winnicottianos, esse texto ainda deixa espaço para aprimorar a abordagem histórica, a análise conceitual, a articulação estrutural, o estudo comparativo, o exame filosófico e a avaliação crítica do léxico winnicottiano considerado no seu todo.

Temos hoje uma concepção mais clara da abrangência das mudanças introduzidas por Winnicott e da unidade interna da sua psicanálise. Dados pessoais, profissionais e institucionais, os quais, como sabemos, são relevantes para a compreensão do desenvolvimento das ideias winnicottianas, e que receberam, no dicionário de J. Abram, uma atenção meramente casual, poderão ser adicionados com proveito. Na segunda edição (2007), a autora adere (ver o capítulo IV) à tese de uma mudança paradigmática no sentido de Kuhn, mas não refaz nem aumenta significativamente os verbetes segundo uma reconstrução precisa do surgimento e da estrutura do paradigma winnicottiano.

O livro de A. Newman, anterior ao de J. Abram, Non-compliance in Winnicott's Words, de 1995, não possui, segundo o próprio autor, características de um dicionário 
propriamente dito, sendo antes uma compilação de textos de Winnicott acompanhados de comentários aleatórios, de cunho pessoal.

O Dicionário Winnicott pretende preencher essa lacuna na literatura sobre Winnicott. O principal resultado esperado é fazer uma reconstrução do léxico do paradigma winnicottiano que seja, na medida do possível, completa, no sentido de analisar a psicanálise winnicottiana de modo abrangente e unificado a partir da totalidade de sua obra e da unidade de seu pensamento teórico-clínico e, igualmente, à luz da teoria kuhniana dos paradigmas e das revoluções científicas. Cuidados especiais serão tomados para preservar as características básicas da linguagem de Winnicott, de sorte que o Dicionário - objetivando a explicitação, ao mesmo tempo histórica, conceitual, estrutural, comparativa, filosófica, crítica e factual do uso e das modificações do uso winnicottiano de termos e de expressões - não resulte no congelamento desses termos na forma de um jargão, mas, por considerar seus sentidos historicamente e, portanto, abertos a elaborações adicionais, se constitua em fonte de inspiração para o desenvolvimento do pensamento winnicottiano.

O núcleo central desse léxico refletirá a estrutura da teoria winnicottiana do amadurecimento, espinha dorsal da sua psicanálise, que será reconstruída segundo as regras seguidas por Winnicott, explícita ou implicitamente, na construção de teorias científicas. O léxico terá ainda uma parte crítica, na qual a psicanálise winnicottiana será avaliada do ponto de vista epistemológico e metodológico. Serão postas em destaque também as possibilidades de uso do pensamento winnicottiano como guia tanto na clínica psicanalítica como na realização de intervenções em diferentes outras áreas, tais como prevenção de distúrbios maturacionais, individuais e sociais, pediatria e obstetrícia, fisioterapia, tratamento de deficiências físicas, assistência social e educação.

De acordo com essa abordagem e baseado nos resultados mencionados da Escola Winnicottiana de São Paulo, será feita a reconstrução da linguagem de Winnicott, traçando seu modo de usar as palavras e, de um modo geral, a relevância das suas teses para uma teoria psicanalítica da vida pessoal e cultural. Atenção especial será dada aos termos novos introduzidos por Winnicott, específicos da sua teoria do amadurecimento (cuidado, dependência, integração, mãe suficientemente boa, continuidade de ser, provisão ambiental, elaboração imaginativa, estrutura da personalidade, identificação cruzada etc.), da psicopatologia (cisão advinda da quebra da linha do ser, agonias impensáveis, retraimento, regressão à dependência, tendência antissocial, falso si-mesmo, psicopatia) e da sociopatologia (não tolerância da ambivalência, tendência 
antidemocrática, pobreza cultural, anarquismo, autoritarismo, beligerância), bem como aos termos da psicanálise tradicional redefinidos (psicose, ambivalência, neurose, instinto, ego, interpretação), postos fora de uso ou mesmo abandonados (pulsão de vida, pulsão de morte, aparelho psíquico) e às palavras emprestadas do inglês cotidiano, tais como self, mutuality, I AM, concern e ruthlessness.

Propondo-se a fazer uma reconstrução abrangente e unificada do léxico da linguagem da psicanálise winnicottiana, com a devida atenção para o seu uso, o Dicionário tem ainda por objetivo adicional elaborar outros tipos de verbetes, divididos em várias outras categorias, que ajudem na reconstituição do ambiente histórico, profissional, institucional e pessoal no qual surgiu a obra de Winnicott.

A realização da tarefa, que segue e amplia a do Dicionário de Roudinesco e Plon, contribuirá para a melhor compreensão do progresso da psicanálise que resultou da revolução winnicottiana e para a determinação do lugar da psicanálise winnicottiana nas ciências do homem, além de facilitar a sua aplicação em diferentes áreas, em particular o seu uso na prevenção e no atendimento de distúrbios de natureza psíquica e social.

Além de servir de recurso importante para o ensino e favorecer o desenvolvimento de pesquisas sobre Winnicott em âmbito nacional e internacional, o Dicionário Winnicott, elaborado no formato aqui proposto - abrangência de tópicos considerados, caráter coletivo do projeto, forte inserção acadêmica da equipe consolidada e qualificada, publicação mediante recursos da tecnologia atual, abertura para o diálogo com os leitores -, contribuirá, fora da área estrita de estudos winnicottianos, tanto para a historiografia da psicanálise em geral como para a atualização do discurso e das práticas psicanalíticas, pondo em evidência a relevância dessa disciplina para as práticas sociais e culturais da nossa época.

\section{Referências}

Abram, J. (2007). The Language of Winnicott (2. a ed.). London: Karnac.

Abram, J. (2013). Donald Winnicott Today. London: Routledge.

Balint, M. (1992). The Basic Fault. Evenston, Ill.: Northwestern University Press.

(Trabalho original publicado em 1968) 
Bird, A. (2000). Thomas Kuhn. Chesham: Acumen.

Caldwell, L. \& Joyce, A. (Orgs.) (2011). Reading Winnicott. London: Routledge.

Davis, M. \& Wallbridge, D. (1981). Boundary and Space. London: Karnac.

Dias, E. O. (2013). A teoria do amadurecimento de D. W. Winnicott. São Paulo: DWW editorial. (Edição inglesa pela Karnac Books em preparação)

Eshel, O. (2013). Reading Winnicott into nano-psychoanalysis: there's plenty of room at the bottom. Psychoanalytic Inquiry, vol. 33, 36-49.

Green, A. (2013). Potential space in psychoanalysis. The object in the setting. In J. Abram, Donald Winnicott Today (pp. 183-204). London: Routledge. (Trabalho original publicado em 1975)

Greenberg, J. \& Mitchell, S. A. (1983). Object Relations in Psychoanalytic Theory. Cambridge, Mass: Harvard University Press.

Hacking, I. (2012). Introductory Essay. In T. S. Kuhn, The Structure of Scientific Revolutions (pp. VII-XXXVII). Chicago: The University of Chicago Press.

Hodges, J. M. (1989). Reshaping the Psychoanalytic Domain. Berkeley: University of California Press.

Horton, P. C., Gerwith, H. \& Kreutter, K. J. (1988). The Solace Paradigm. Madison, Conn.: International Universities Press.

Kohut, H. (1975). The Restoration of the Self. Chicago: University of Chicago Press.

Kuhn, T. S. (2012). The Structure of Scientific Revolutions. Chicago: The University of Chicago Press. (Trabalho original publicado em 1962) 
Lifton, R. (1976). From Analysis to Formation: Toward a Shift in Psychoanalytic paradigm. Journal of the American Academy of Psychoanalysis, 4, 65-94.

Loparic, Z. (2002). Winnicott's Paradigm Outlined. Revista latinoamericana de psicopatologia fundamental, 5(1), 61-98.

Loparic, Z. (2006). De Freud a Winnicott: aspectos de uma mudança paradigmática. Winnicott e-Prints, 5(2), 1-29.

Loparic, Z. (2013). From Freud to Winnicott: Aspects of a Paradigm Change. In J. Abram, Donald Winnicott Today (pp. 113-156). London: Routledge.

Loparic, Z. (Org.) (2016). Winnicott and the future of psychoanalysis. Proceedings of the First International Winnicott Congress of the International Winnicott Association. São Paulo: DWW editorial. (No prelo)

McDougall, J. (1997). As múltiplas faces de Eros. São Paulo: Martins Fontes.

Mitchell, S. A. (2000). Relationality. Hillsdale, NJ: The Analytic Press.

Modell, A. H. (1968). Object Love and Reality. New York: International Universities Press.

Nickles, T. (Org.) (2003). Thomas Kuhn. Cambridge: Cambridge University Press.

Ogden, T. H. (2002). Reading Winnicott. In T. H. Ogden, Conversations at the Frontier of Dreaming (pp. 203-235). London: Karnac.

Ornstein, P. H. (1992). How to Read "The Basic Fault". In M. Balint, The Basic Fault (pp. VII-XXV). Evenston, Ill.: Northwestern University Press.

Phillips, A. (1988). Winnicott. London: Penguin. 
Roussillon, R. (2010). Winnicott's Deconstruction of Primary Narcissism. In J. Abram, Donald Winnicott Today (pp. 270-290). London: Routledge.

Rycroft, C. (1985). Psychoanalysis and Beyond. London: The Hogarth Press.

Thompson, N. (2013). Winnicott and American Analysts. In J. Abram, Donald Winnicott Today (pp. 386-417). London: Routledge.

Widlöcher, D. (2013). Winnicott and the Acquisition of a Freedom of Thought. In J. Abram, Donald Winnicott Today (pp. 235-269). London: Routledge. (Trabalho original publicado em 2006)

Winnicott, D. W. (1958). Through Paediatrics to Psychoanalysis. London: Karnac.

Wright, K. (2013). The Search for Form. A Winnicottian Theory of Artistic Creation. In J. Abram, Donald Winnicott Today (pp. 250-269). (Trabalho original publicado em 2009) 